\title{
Anaphylactic Shock Caused by Ingestion of Polyethylene Glycol
}

\author{
Sun Hee Lee, Jae Myung Cha, Joung Il Lee, Kwang Ro Joo, Hyun Phil Shin, Il Hyun Baek, Jung Won Jeon, \\ Jun Uk Lim, Jung Lok Lee, Hyae Min Lee, Young-Hak Cho \\ Department of Internal Medicine, Kyung Hee University Hospital at Gang Dong, Kyung Hee University School of Medicine, Seoul, Korea
}

Colonoscopy is the current standard method for evaluation of the colon. The diagnostic accuracy and therapeutic safety of colonoscopy depend on the quality of colonic cleansing and preparation. Generally, all these preparations have been demonstrated to be safe for use in healthy individuals without significant comorbid conditions. Based on safety and efficacy concerns, polyethylene glycol (PEG) is most commonly utilized as a bowel preparation solution for colonoscopy. Adverse events in patients receiving PEG are mostly clinically non-significant. However, fatal adverse events rarely have been shown to occur in the few individuals who experience vomiting or aspiration. Anaphylactic shock associated with ingestion of PEG electrolyte solution is an extremely rare fatal complication, and reported mainly in Western countries. Here, we report the first case of anaphylactic shock following the ingestion of PEG solution in Korea. (Intest Res 2015;13:90-94)

Key Words: Polyethylene glycols; Anaphylaxis; Adverse effects; Colonoscopy; Shock

\section{INTRODUCTION}

Recently, the incidence of colorectal cancer (CRC) has drastically increased in South Korea, and colonoscopy is being recommended for screening of CRC in adults aged over 50 years. ${ }^{1}$ Although appropriate colonic cleansing is essential prior to colonoscopy, effective, safe, easy-to-administer, and contraindication-free colonic cleansing agents have not yet been developed. As colonoscopy has become a common examination method, the use of colonic cleansing agents is also increasing, as expected, warranting better understanding and proper management of their side effects. ${ }^{2}$

Liquid sodium phosphate as a bowel purgative has been widely used owing to its ease of administration, but is not prescribed anymore because of the risk of renal failure. ${ }^{3}$

Received April 4, 2014. Revised June 18, 2014. Accepted July 11, 2014. Correspondence to Jae Myung Cha, Department of Internal Medicine, Gand Dong Kyung Hee University Hospital, 892 Dongnam-ro, Gangdong-gu, Seoul 134-727, Korea. Tel: +82-2-440-6113, Fax: +82-2-440-6295, E-mail: drcha@khu.ac.kr

Financial support: None. Conflict of interest: None.
Most colonic cleansing agents are based on polyethylene glycol (PEG), which does not cause translocation of electrolytes and water as it penetrates the intestine without inducing absorption and secretion, and is hence considered relatively safe.,4 Patients, however, still complain of nausea, vomiting, abdominal pain, abdominal bloating, chest pains, and dizziness in some cases. ${ }^{2,4}$ Most side effects are minor, but there are several reported cases of fatal side effects resulted from vomiting and aspiration during PEG administration, i.e., Mallory-Weiss syndrome, ${ }^{5}$ esophageal rupture, ${ }^{6}$ aspiration pneumonia, and acute respiratory distress syndrome. ${ }^{7}$ Furthermore, there are a few case reports regarding hypersensitivity as a side effects of PEG agents, ${ }^{8-11}$ as well as anaphylaxis, which can in some cases be fatal. ${ }^{12-14}$ However, no case report on anaphylaxis associated with ingestion of PEG has been published in South Korea.

In the current study, we report of a 39-year-old man who experienced anaphylactic shock due to PEG ingestion prescribed for colonoscopy. 


\section{CASE REPORT}

A 39-year-old male office worker without a previous history of specific diseases and symptoms underwent a scheduled colonoscopy as part of a medical check-up. The patient underwent colonoscopy in another institute 3 years ago, and did not experience any specific symptoms from the use of sodium phosphate bowel purgative (Solin oral solution, Korea Pharma Co. Ltd., Seoul, Korea) as a colonic cleansing agent. Other examinations also indicated no abnormal findings.

One day prior to the colonoscopy, the patient received a dose of PEG agent (Colyte powder, Taejoon Pharma Co. Ltd., Seoul, Korea). About 5 minutes later, he experienced a generalized rash, itching, swelling and tingling feeling in his arms, dizziness, dysphagia, and dyspnea, and therefore, he visited our emergency room (ER). The patient did not have a history of chronic diseases (e.g., diabetes, hypertension, tuberculosis, and hepatitis), or any allergic disorders such as bronchial asthma and food allergies. He had been smoking for over 10 years and drinking at least once per week. In addition, no specific family history was noted, including any known occurrence of CRC.

Within $30 \mathrm{~min}$, the patient was transferred to the ER by ambulance. When admitted to the ER, he was in a state of shock, with a blood pressure of $41 / 31 \mathrm{mmHg}$, heart rate of 102 beats/min, respiratory rate of 28 breaths/min, and body temperature of $36.0^{\circ} \mathrm{C}$. Even though the patient showed acute symptoms, he was still conscious. No specific findings were noted on chest and abdominal examinations. The liver and spleen were not palpable. Blood tests revealed leukocytes, hemoglobin, and platelet levels of $6,700 / \mathrm{mm}^{3}(92.9 \%$ neutrophils, and $0.0 \%$ eosinophils), $15.5 \mathrm{~g} / \mathrm{dL}$, and 238,000/ $\mathrm{mm}^{3}$, respectively, which were all within normal ranges. Electrolyte tests showed that sodium, potassium, and chloride levels were $141 \mathrm{mEq} / \mathrm{L}, 3.1 \mathrm{mEq} / \mathrm{L}$, and $106 \mathrm{mEq} / \mathrm{L}$, respectively.

Other results on biochemical and urine analyses were found to be within normal ranges. In arterial blood gas analysis, levels of $\mathrm{pH}, \mathrm{pCO}_{2}, \mathrm{pO}_{2}$, and bicarbonate were 7.427, 35.2 $\mathrm{mmHg}, 84.0 \mathrm{mmHg}$, and $23.2 \mathrm{mEq} / \mathrm{L}$, respectively. Cardiac enzyme tests revealed a CK-MB level of $0.7 \mathrm{ng} / \mathrm{mL}$ and a Troponin I level of $0.012 \mathrm{ng} / \mathrm{mL}$, both of which were within normal ranges. The heart rate was found to be 82 regular sinus rhythms per minute on electrocardiography, and no significant findings were revealed on chest and abdominal radiography. The patient was interviewed completely and thoroughly several times regarding food allergens, medica- tions, exercise, and occupational exposure to hazardous substances that can cause anaphylaxis. However, no cause other than the administration of the PEG agent could be identified.

As anaphylactic shock by PEG was suspected, oxygen was supplied via a nasal cannula at a rate of $4 \mathrm{~L} / \mathrm{min}$. Although the patient was administered with $1 \mathrm{~L}$ of saline as well as an intravenous injection of $0.1 \mathrm{mg}$ of epinephrine (epinephrine injection solution, Daihan Pharm Co., Seoul, Korea), $3 \mathrm{mg}$ of piprinhydrinate (Plakon ${ }^{\circledR}$, Yungjin Pharm Co., Seoul, Korea), and $125 \mathrm{mg}$ of methylprednisolone (Salon ${ }^{\circledR}$, Hanlim Pharm Co., Seoul, Korea), his hypotension was not alleviated after 10 minutes. Therefore, $1 \mathrm{mg}$ of epinephrine was dissolved in $0.5 \mathrm{~L}$ of glucose injection solution $(5 \%)$ and then injected with the constant rate of $1 \mu \mathrm{g} / \mathrm{min}$. Most symptoms (e.g., difficulty in breathing, hives, itching, and other symptoms) improved 1 hour after treatment, and vital signs returned to normal (blood pressure, 120/78 $\mathrm{mmHg}$; heart rate, 92 beats/min; respiratory rate, 20 breaths $/ \mathrm{min}$ ). The continuous injection of epinephrine and oxygen supply were subsequently discontinued. In order to confirm whether the PEG agent was responsible for the anaphylaxis, a skin prick test was planned but not performed because the patient firmly refused to undergo the test owing to a fear of experiencing another anaphylactic shock.

Three hours after admission to the ER, the patient only showed edema on both arms, but all other symptoms were attenuated or they disappeared. After 20 hours, his vital signs were stable and no further evidence of exacerbation and recurrence were noted. The patient was informed about the possibility of anaphylaxis caused by the PEG agent, and advised to avoid such substances in the future. After discharge, no evidence of recurrence or after-effects was observed on subsequent outpatient follow-ups.

\section{DISCUSSION}

PEG electrolyte solutions have been used since 1980 for colonic cleansing, and are still widely utilized nowadays. These solutions replace chloride ions, which are required for active absorption of sodium ions in the colonic lumen, with sulfate ions, thereby minimizing their absorption. Further, high molecular weight PEG $(3,350 \mathrm{~g} / \mathrm{mol}$ or $4,000 \mathrm{~g} / \mathrm{mol})$ is added to the solution to hinder water absorption given the lower molecular weight of sulfate ions compared to that of sulfate ions. ${ }^{15-17}$ In the present case report, the PEG solution contained PEG 3350 (236 g), sodium chloride (5.86 g), sodium bicarbonate ( $6.74 \mathrm{~g}$ ), potassium chloride ( $2.97 \mathrm{~g}$ ), and anhydrous sodium sulfate (22.74 g). This PEG electrolyte so- 
lution is known to be safe because it maintains the osmotic pressure similar to that of blood plasma, and does not cause translocation of electrolytes and water by penetrating the intestine and preventing absorption and secretion. PEG solution reduces the toxicity of proteins and other substances, extends the half-life of other drugs, and is chemically and immunologically inert, and hence, considered safe. ${ }^{13}$ Various molecular weights of PEG have been widely utilized for numerous applications such as injection solutions, pills, aqueous solutions, skin disinfectants, and toothpastes. However, several cases of allergic reactions have been reported.

Anaphylaxis occurs immediately after exposure to a specific sensitized antigen and may lead to death in severe cases. ${ }^{18}$ The most common causes include food items, insect stings, and drugs, but non-immunological stimuli such as exercise and extreme environmental conditions (cold or hot condition) rarely contribute to anaphylaxis; however the causative mechanism has not been established clearly. ${ }^{19}$ Anaphylaxis is diagnosed when more than two of the following symptoms are observed within several minutes to hours after exposure to a suspected antigen: invasion to mucocutaneous tissues, respiratory symptoms, hypotension, and persistent digestive symp toms. ${ }^{18}$ In the current case, the patient was diagnosed with anaphylaxis because of immediate findings of skin rash, itching, dyspnea, and hypotension 5 minutes after PEG exposure.

Since 1990, there have been 7 cases regarding allergic reactions and anaphylaxis caused by the administration of PEG electrolyte solution reported in the literature (Table 1) ${ }^{8-14} \mathrm{In}$ these cases, the mean patient age was 58.4 years, and 4 of the 7 patients were men. As shown in Table 1, only 2 cases showed anaphylaxis shock with associated hypotension. ${ }^{12,13}$ In the case of Schuman et al., ${ }^{12}$ the patient complained of dyspnea (stridor) during medication and had hypotension (80 mmHg systolic blood pressure) 20 minutes after admission. The symptoms were alleviated after the administration of epinephrine.

Similarly, in the case report by Shah et al. ${ }^{13}$ the patients presented with hypotension, loss of consciousness, dyspnea, and hives, all of which improved with epinephrine administration. This patient underwent a skin prick test using PEGs with 200, 1000, 3350, and 9000 molecular weights, and had positive reactions against PEGs with 3350 and 9000 . The patient experienced a skin rash and swelling when using PEGcontaining sun block and toothpaste. In addition, this patient had allergic symptoms (e.g., hives, dyspnea, itching) after taking pills containing PEGs, which were attenuated by selfinjection of epinephrine.

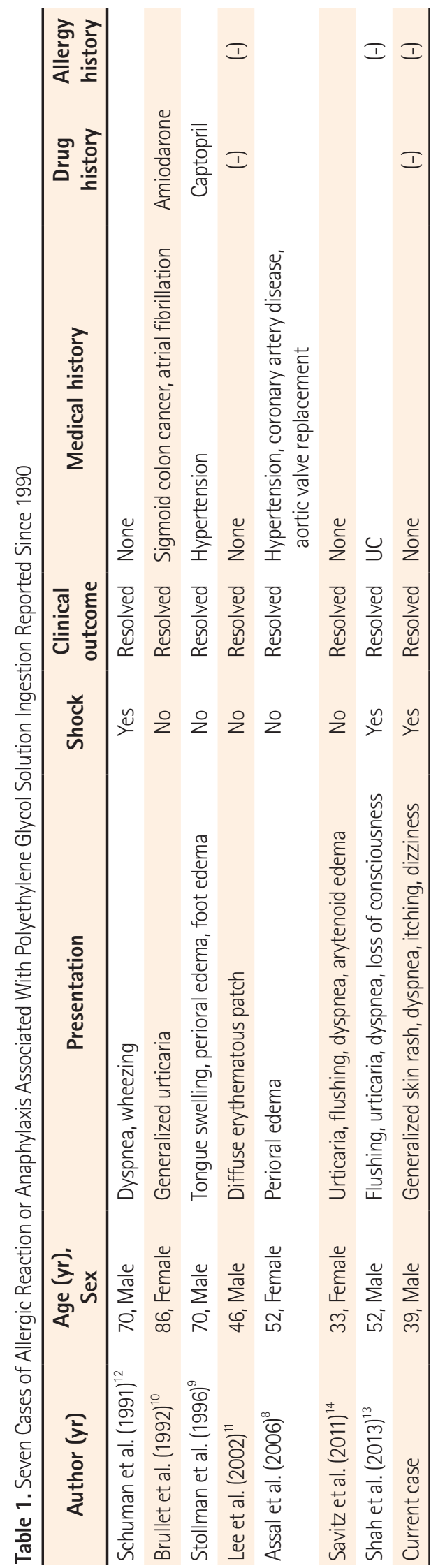


Another male patient who showed allergic reactions against a PEG-containing povidone-iodine disinfection gel showed the same symptoms for shampoos and colloid solutions containing PEG on skin prick tests, thus confirming his positive reactions against PEGs and their analogs. Therefore, it is essential to perform skin prick tests for not only PEGs with various molecular weights but also their analogs for patients with positive reactions against PEG, thereby educating patients to avoid potential exposures to such substances.

In the case report by Savits et al. ${ }^{14}$ the patient complained of hives, dyspnea, and a sensation of throat-closing after the administration of a PEG agent; the symptoms were alleviated with administration of anti-histamines and steroids, but the tongue swelling worsened 20 hours later, causing the patient to re-visit the ER. The symptoms improved with epinephrine treatment. Approximately 1-20\% patients who experienced anaphylaxis may experience secondary reactions within 1-72 hours, however, there is no reliable indicator to predict such recurrences. ${ }^{18}$ Therefore, it might be appropriate for doctors to carefully examine secondary reactions even after alleviation of such symptoms in patients with anaphylaxis caused by the administration of PEG agents.

As in the current case, epinephrine has often been selected as a therapeutic option for patients with anaphylaxis. However, great caution is required as excessive administration may result in side effects including ventricular arrhythmias, angina pectoris, myocardial infarction, pulmonary edema, and hemorrhage. ${ }^{20}$ Generally, 1:1,000 diluted epinephrine is intramuscularly delivered every $5-15$ minutes, $0.2-0.5 \mathrm{mg}$ per injection. However, continuous jugular vein injections have been recommended, in which appropriate fluid therapy along with epinephrine injection was not found to alleviate hypotension or result in cardiac arrest. ${ }^{18}$ In anaphylaxis patients with hypotension, intravenous injection of epinephrine is considered to be safe and effective, but no direct comparison has yet been made to muscular injections. ${ }^{21}$ The continuous injection of epinephrine is beneficial because it is easy to control the dose until a certain potency is achieved, whilst bolus injections have risks of overdose, and simultaneous cardiovascular monitoring should therefore be considered.$^{18}$ In our case, a combination of fluid therapy and intravenous epinephrine injection was provided because the patient was already in state of shock when admitted to the ER.

In cases reported by Assal et al. ${ }^{8}$ and Stollman et al., ${ }^{9}$ angioedema was observed without other symptoms. However, Brullet et al. ${ }^{10}$ reported that the patient presented with generalized itching and hives. In these cases, all the symptoms improved with antihistamine and steroid treatment. Lee et al. ${ }^{11}$ reported that treatment using antihistamine and steroids was only successful for patients with truncal rashes caused by the PEG agent. In many case reports, skin lesions and dyspnea were the most common symptoms, and appropriate medical treatments improved such symptoms without further complications as in the current case, indicating that immediate therapeutic treatment provides favorable prognoses.

Although histamine and tryptase levels increase in the blood and accompany type I hypersensitivity indicating allergic reactions, they produce high rates of false-negatives and are therefore limited as diagnostic tests. ${ }^{18}$ Skin prick and serum specific IgE tests are common diagnostic tools to diagnose allergic diseases and to confirm allergens. The serum specific IgE test is easy and safe to perform but is prone to low sensitivity and a high likelihood of detecting IgE antibodies associated with cross-reactions. ${ }^{22}$ Results of these examinations are usually relatively consistent, although in some cases this is not the case, and simultaneous examinations are therefore warranted. Skin prick tests examine various antigens at the same time and provide results immediately. However, they could be dangerous for patients with a history of anaphylactic shock, such as in the present case. Oral provocation tests, mostly used for foods allergen diagnosis, are not frequently performed because these can also be potentially dangerous in patients with anaphylactic shock. ${ }^{23}$ Sohy et al. ${ }^{24}$ implemented the skin prick and oral provocation test by using PEG 4000 to elucidate the causative mechanisms of anaphylaxis. However, such confirmatory tests were not performed, and this represents a limitation of the case.

The current study is the first report in South Korea describing anaphylactic shock caused by ingestion of PEG, which is generally considered safe. Although it is rare, physicians must be cautious of this potentially fatal complication when administering PEG agents to patients undergoing colonoscopy.

\section{REFERENCES}

1. Lee BI, Hong SP, Kim SE, et al. Korean guidelines for colorectal cancer screening and polyp detection. Intest Res 2012;10:67-88.

2. Wexner SD, Beck DE, Baron TH, et al. A consensus document on bowel preparation before colonoscopy: prepared by a task force from the American Society of Colon and Rectal Surgeons (ASCRS), the American Society for Gastrointestinal Endoscopy (ASGE), and the Society of American Gastrointestinal and Endoscopic Surgeons (SAGES). Gastrointest Endosc 2006;63:894909. 
3. Markowitz GS, Stokes MB, Radhakrishnan J, D’Agati VD. Acute phosphate nephropathy following oral sodium phosphate bowel purgative: an underrecognized cause of chronic renal failure. J Am Soc Nephrol 2005;16:3389-3396.

4. Rostom A, Jolicoeur E, Dubé C, et al. A randomized prospective trial comparing different regimens of oral sodium phosphate and polyethylene glycol-based lavage solution in the preparation of patients for colonoscopy. Gastrointest Endosc 2006;64: 544-552.

5. Raymond PL. Mallory-Weiss tear associated with polyethylene glycol electrolyte lavage solution. Gastrointest Endosc 1991;37: 410-411.

6. Kim CH, Park JH, Kim MW, et al. A case of Boerhaave's syndrome after bowel preparation with polyethylene glycol. Korean J Gastrointest Endosc 2005;31:315-319.

7. de Graaf P, Slagt C, de Graaf JL, Loffeld RJ. Fatal aspiration of polyethylene glycol solution. Neth J Med 2006;64:196-198.

8. Assal C, Watson PY. Angioedema as a hypersensitivity reaction to polyethylene glycol oral electrolyte solution. Gastrointest Endosc 2006;64:294-295.

9. Stollman N, Manten HD. Angioedema from oral polyethylene glycol electrolyte lavage solution. Gastrointest Endosc 1996;44: 209-210.

10. Brullet E, Moron A, Calvet X, Frias C, Sola J. Urticarial reaction to oral polyethylene glycol electrolyte lavage solution. Gastrointest Endosc 1992;38:400-401.

11. Lee JS, Chae HS, Chung WC, et al. Urticaria reaction by oral polyethylene glycol ingestion. Korean J Gastrointest Endosc 2002;24:299-301.

12. Schuman E, Balsam PE. Probable anaphylactic reaction to polyethylene glycol electrolyte lavage solution. Gastrointest Endosc 1991;37:411.

13. Shah S, Prematta T, Adkinson NF, Ishmael FT. Hypersensitivity to polyethylene glycols. J Clin Pharmacol 2013;53:352-355.
14. Savitz JA, Durning SJ. A rare case of anaphylaxis to bowel prep: a case report and review of the literature. Mil Med 2011;176:944945.

15. Davis GR, Santa Ana CA, Morawski SG, Fordtran JS. Development of a lavage solution associated with minimal water and electrolyte absorption or secretion. Gastroenterology 1980;78: 991-995.

16. Hyun JJ, Jeen YT, Park SH, et al. Comparison of 4 L of PEG versus combination of $2 \mathrm{~L}$ of PEG and $45 \mathrm{~mL}$ of sodium phosphate for colonoscopy colon cleansing: a prospective randomized trial. Korean J Gastrointest Endosc 2005;31:368-373.

17. Yamasuji Y, Higashi Y, Sakanoue M, et al. A case of anaphylaxis caused by polyethylene glycol analogues. Contact Dermatitis 2013;69:183-185.

18. Sampson HA, Muñoz-Furlong A, Campbell RL, et al. Second symposium on the definition and management of anaphylaxis: summary report--Second National Institute of Allergy and Infectious Disease/Food Allergy and Anaphylaxis Network symposium. J Allergy Clin Immunol 2006;117:391-397.

19. Worm M, Babina M, Hompes S. Causes and risk factors for anaphylaxis. J Dtsch Dermatol Ges 2013;11:44-50.

20. Kemp SF, Lockey RF, Simons FE. Epinephrine: the drug of choice for anaphylaxis. A statement of the World Allergy Organization. Allergy 2008;63:1061-1070.

21. Brown SG, Blackman KE, Stenlake V, Heddle RJ. Insect sting anaphylaxis; prospective evaluation of treatment with intravenous adrenaline and volume resuscitation. Emerg Med J 2004; 21:149-154.

22. Chung HL. Clinical significance of serum IgE. Korean J Pediatr 2007;50:416-421.

23. Ito K, Urisu A. Diagnosis of food allergy based on oral food challenge test. Allergol Int 2009;58:467-474.

24. Sohy C, Vandenplas O, Sibille Y. Usefulness of oral macrogol challenge in anaphylaxis after intra-articular injection of corticosteroid preparation. Allergy 2008;63:478-479. 